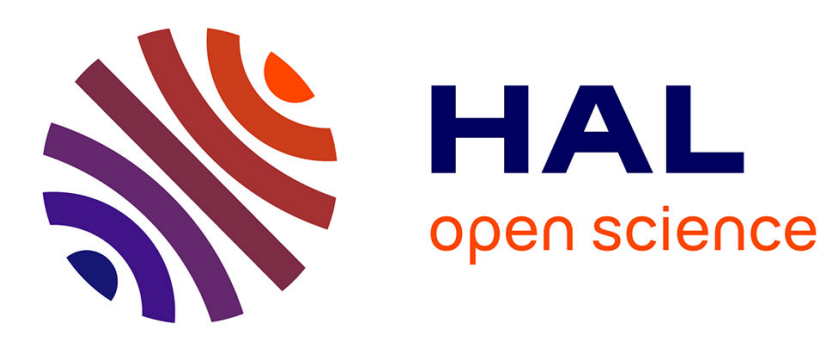

\title{
Interactive Cell Injection Simulation Based on 3D Biomechanical Tensegrity Model
}

\author{
Hamid Ladjal, Jean-Luc Hanus, Antoine Ferreira
}

\section{To cite this version:}

Hamid Ladjal, Jean-Luc Hanus, Antoine Ferreira. Interactive Cell Injection Simulation Based on 3D Biomechanical Tensegrity Model. IEEE/RSJ International Conference on Intelligent Robots and Systems., Sep 2008, Inconnu, France. pp.2296 - 2302, 30, 10.1109/IROS.2008.4650973 hal-00653111

\section{HAL Id: hal-00653111 \\ https://hal.science/hal-00653111}

Submitted on 17 Dec 2011

HAL is a multi-disciplinary open access archive for the deposit and dissemination of scientific research documents, whether they are published or not. The documents may come from teaching and research institutions in France or abroad, or from public or private research centers.
L'archive ouverte pluridisciplinaire HAL, est destinée au dépôt et à la diffusion de documents scientifiques de niveau recherche, publiés ou non, émanant des établissements d'enseignement et de recherche français ou étrangers, des laboratoires publics ou privés. 


\title{
Interactive Cell Injection Simulation Based on 3D Biomechanical Tensegrity Model
}

\author{
Hamid Ladjal, Jean-Luc Hanus and Antoine Ferreira
}

\begin{abstract}
To facilitate training of biological cell injection operations, we are developing an interactive virtual environment to simulate needle insertion into biological cells. A key challenge of deformable simulation is to satisfy the conflicting requirements of real-time interactivity and physical realism. This paper presents methodologies for dynamic modeling, visual/haptic display and model validation of cell injection. We first investigate the challenging issues in the modeling of the bio-mechanical properties of living cells. We propose a dynamic model to simulate cell deformation and puncture. The developped approach is based on the assumptions that the mechanical response of living cells is mainly determined by the cytoskeleton and that the cytoskeleton is organised as a tensegrity structure including microfilaments, microtubules and intermediate filaments. Equivalent microtubules struts are represented with a linear mass-tensor finite element model and equivalent microfilaments and intermediate filaments with viscoelastic Kelvin-Voigt elements. The virtual environment has been implemented with both graphic and haptic interfaces.
\end{abstract}

\section{INTRODUCTION}

Living cells are increasingly used in drug discovery, functional genomics and toxicology to name a few. In fact, biological cell micromanipulations are currently performed manually and efficiency is relatively low depending strongly on the experience of the operator. A review on the existing techniques for manipulating biological cells is presented in [6]. The intracytoplasmic sperm injection process (ICSI) results in low success rate and poor reproducibility [7], [8]. Knowledge of interactive forces during pipette insertion plays an important role in precise micro injection operation [29]. This knowledge can help to identify and model different tissue types and it can also provide feedback for precise control of robot-assisted insertion while reducing tissue deformation and needle deflection [1]. However, when manipulating deformable biological objects, force sensor measurement will provide only the local forces at the pipette puncture point which limits strongly the operator haptic rendering [32]. In order to learn, train and analyze basic cell injection procedures, most operators are aiming to use artificial cells because practicing on human oocyte has ethical concerns and potential risks. This work is intended to propose a 3D pseudo-haptic rendering of biological cells for injection assistance. Similar research on virtual reality environments platforms have been carried out at a macroscopic scale in surgery simulation and modeling of soft tissues [5], [9],

This work was funded by the Centre de Recherche en Biologie (CER) of Baugy and supported by the Laboratoire de Biologie d'Aide à la Procréation of the Hôpitaux de Paris-Pitié Salpétrière

H. Ladjal, J-L Hanus and A.Ferreira are with the PRISME Institute, ENSI Bourges, University of Orléans, 18000 Bourges, France antoine.ferreira@ensi-bourges.fr

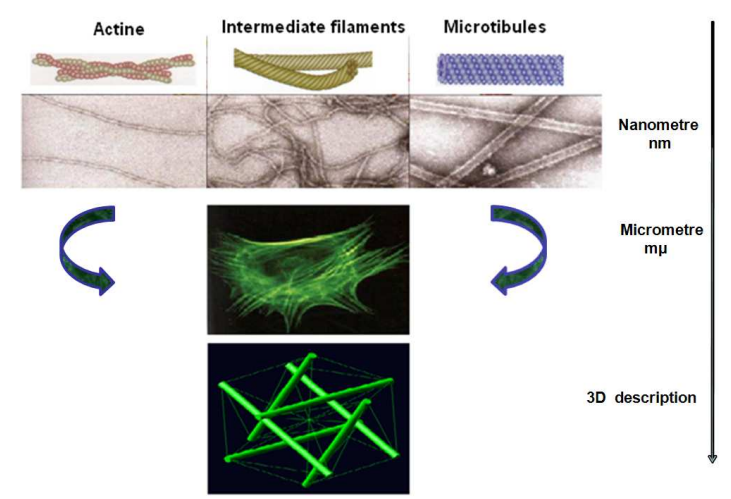

Fig. 1. Cytoskeleton and equivalent biomechanical tensegrity model

[10], [21]. The needle punctures and passes through different tissue layers such as skin, muscle, fatty and connective tissue [33],[34]. By analogy to in - vivo intracytoplasmic sperm injection, similar boundary conditions occurs. The cell geometry and biomechanical tissue properties (biomembrane, cytoplasm, nucleus) are of major importance in simulation and modeling because these factors affect the amount of cell membrane deformation, needle deflection and interaction forces. It requires a nonlinear biomechanical cell model able to render in a realistic way the interactions between the needle and the cell. Knowing interaction forces and developing appropriate pipette deflection and tissue deformation models during pipette insertion are the key issues for accurate insertion. In this study, we first investigate the challenging issues in the modeling of the biomechanical properties of living cells. Then we propose a dynamic model, based on an equivalent cystoskeleton, to simulate cell deformation during punction.

The developped approach is based on two main assumptions:

- the mechanical response of living cells is mainly determined by the cytoskeleton;

- the cytoskeleton is organised as a tensegrity structure including microfilaments, microtubules and intermediate filaments.

A simplified cytoskelton is developped with microtubules struts represented with a linear mass-tensor finite element model and microfilaments and intermediate filaments with viscoelastic Kelvin-Voigt elements. Both graphic and haptic rendering are provided in real-time to the operator through a $3 \mathrm{D}$ virtual environment.

This paper is organized as follows. In Section II, we present an overview of the mechanical models for living cells 


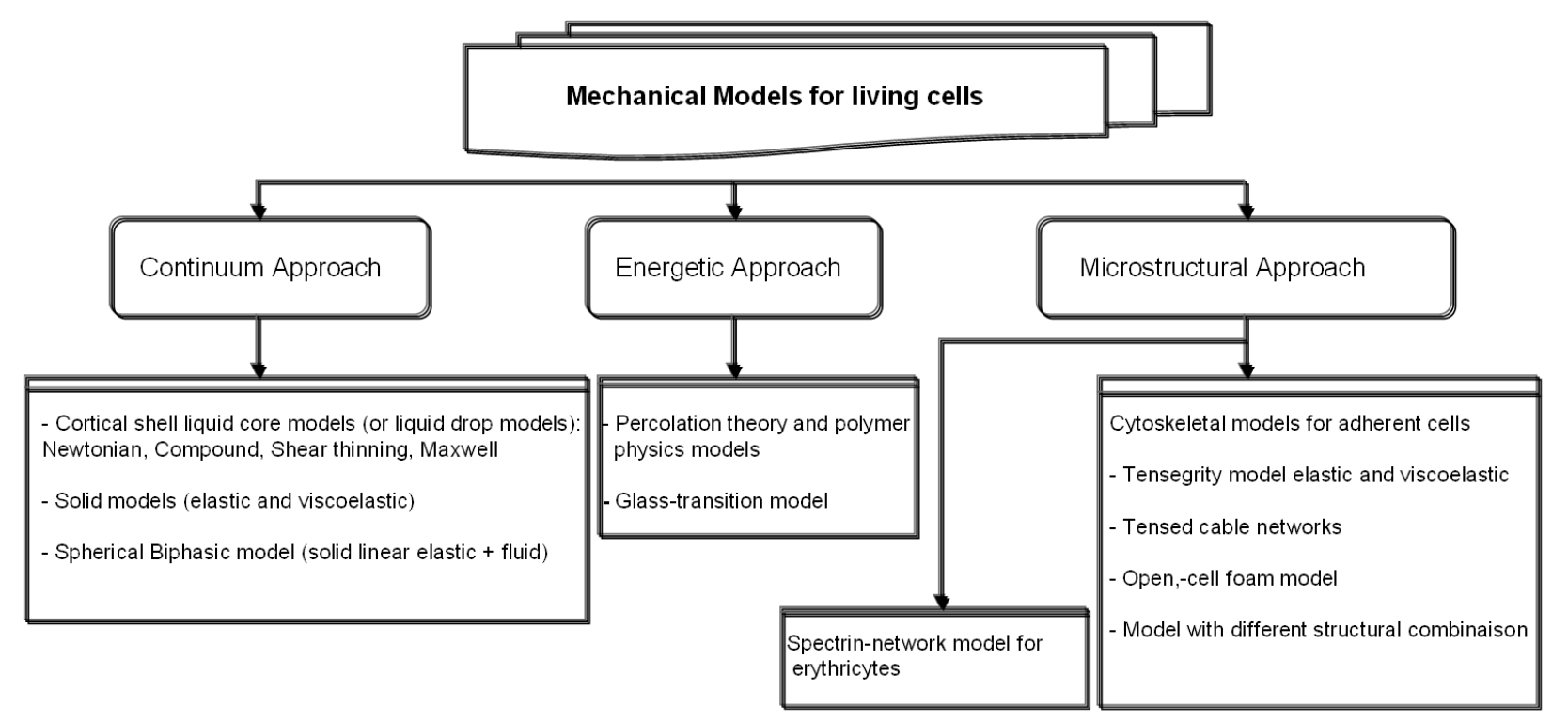

Fig. 2. An overview of the mechanical models of living cell: Three principal classes (continuum, energetic and structural approaches).

and the adopted tensegrity model for cell modeling. In Section III, we present the different deformation algorithms of soft tissue before to define a model for real-time simulation. In Section IV, we present the software architecture of the real-time 3D pseudo-haptic rendering simulation platform. In Section V, we present simulation results.

\section{BIOMECHANICAL MODELS FOR BIOLOGICAL CELLS}

\section{A. Related Research}

In this section, we review major biomechanical models of the living cells as shown in Fig. 2. The development of biological cell models able to simulate insertion forces such as the force peak, latency in the force changes, and separation of different forces such as stiffness and damping force is a challenging issue. The majority of the proposed models are mainly derived into three classes: continuum, energetic and structural approaches. The models belonging to the first class assumes the biological cell comprising materials with certain continuum material properties without any insights on molecular mechanical properties cortical shell liquid core models [2], solid models [3] or spherical biphasic models [4]. For further explanation on these models, the reader may refer to [16]. Their main advantages are their facility to compute the mechanical properties of cells and provides details on the distribution of stresses and strains induced on cells. However, this macroscopic continuum approach, which views "the cell has a tensed balloon filled with molasses or jello [14]", has its drawbacks since it is nor capable of accounting for the molecular deformations and interactions within the cell.

The second category of models takes into account the contributions of various cytoskeleton structures to the overall energy budget of cell during contraction. It is based on the percolation theory and polymer physics models at large deformations. The advantage of this model is its independence in the choice of its coordinate system and the particular details of the cytoskeleton architecture, because the energy is a scalar quantity. However, its implementation requires the assumption of large deformations of the cell.

The third class of models includes the tensegrity structures divided into two subclasses:spectrin-network model and cytoskeletal models for adherent cells. The former deals a specific microstructural network for spectrin cells at large deformations [14]. The cytoskeleton as the main structural component and attribute a central role to cytoskeleton contractile forces. Recently, a specific architectural model of the cytoskeletal framework (tensegrity) deserved wide attention. The tensegrity approach has described many aspects of cell deformability including non-linear features of cellular structural behavior [17][14]. These models view the cell as a network of microfilament, microtubule and actin, that distribute forces within the cell through a balance of compression and tension [16][14]. Recent studies show that the tensegrity structure used with viscoelastic elements as a model of the cytoskeleton allows an accurate analysis of living cells behavior [20].

In this study, we show that the micro/nanostructural behavior of an oocyte cell composed of multilayer material components such as the pellucida zona, the biomembrane and the cytoplasm cytoskeleton could be modeled by the tensegrity model coupled to a viscoelastic membrane (see Fig.3).

\section{B. Tensegrity model}

The overall structure of the tensegrity model is described as follows. The cytoskeleton is composed of an interconnected structure of various cross-linked and interlinked filamentous biopolymers that extends from the center to the cell surface (bio-membrane). Three major filamentous components composes the cytoskeleton, i.e. the actin microfilaments, microtubules and intermediate filaments, and 

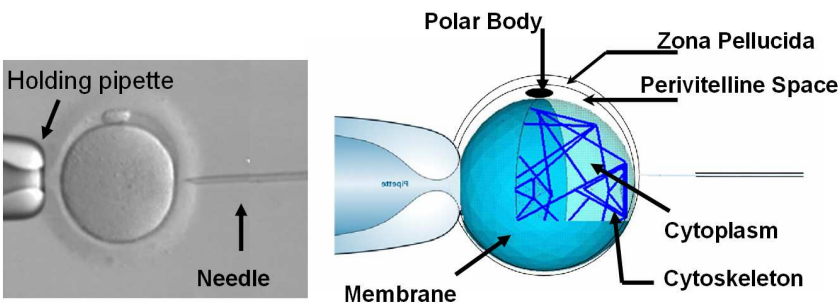

Fig. 3. (a) Biological oocyte cell for micro injection procedure and (b) tensegrity model.

are physically interlinked. The following section studies the mechanical properties and geometrical structure of the tensegrity model.

1) Mechanical Properties: Fig.4 presents the mechanical characteristics of the filamentous components when exposed to shearing force. The results show that the actins and microtubules act as tendons with different rupture characteristics, i.e., high force-small displacement or small force-high displacement, respectively. Compared to actin and microtubule filaments, the intermediate filaments are contrasting. They behave as a stiff spring supporting important tension and stretching forces without mechanical rupture. they are well adapt at maintain the shape cell [31].

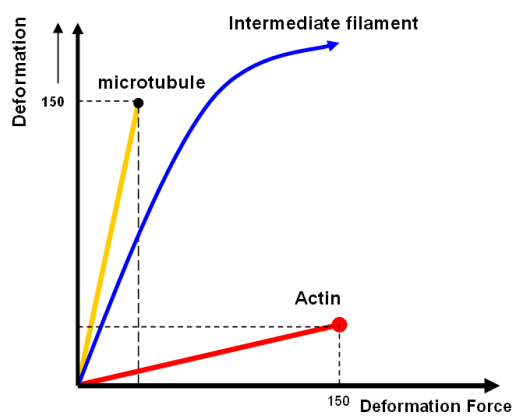

Fig. 4. Mechanical Properties of the actin microfilaments and microtubules and filaments intermediate [31]

2) Geometry of the simplified tensegrity model: In our simulation, we choose a simplified tensegrity structure with six compressing struts (two in each orthogonal direction), these struts aggregate behavior of the microtubules, as viewed as beams as shown in Fig.5. These struts are attached to 36 cable segments [?] :

- 12 cables representing the intermediate filaments which are connected to the parallel struts.

- 24 cables representing the actin microfilaments connected to the end points of each strut.

\section{FINITE ELEMENT MODELING}

\section{A. Related work in FEM Simulation}

Soft tissue modeling are usually derived using either massspring approach or Finite Element Method (FEM). Massspring models are less "physically based". They comprise a set of nodes connected by springs, with point masses attached at each node. Real time performance can be achieved with a

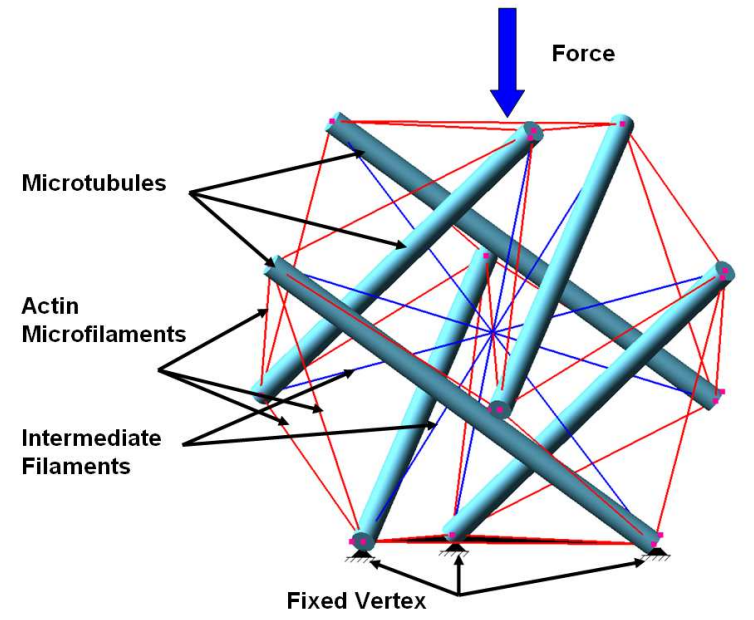

Fig. 5. Tensegrity model of the cell.

limited number of nodes but the behavior is often unrealistic and can be unstable. The FEM approach produces more accurate results but requires high computational costs for realtime modeling. Much research effort has been concentrated on developing techniques to speeding up the finite element computation of deformable tissues without lack of accuracy. In [22], the authors modeled the viscoelastic behavior of the tissue by assuming that the elastic deformation is linear. In [23], the authors introduce a robust adaptive method for simulating dynamic deformations of a viscoelastic object in real-time using an explicit finite-element method. The works presented in [24][25][26] use a deformable model based on finite element nonlinear mass-tensor formalism using Venant-Kirchoff elastic model for large displacements or Kelvin-Voigt viscoelastic element. In this study, we propose a hybrid model well adapted to the deformable microstructural geometry of the oocyte cell. The biomechanical model we used is a simplified cyctoskeleton tensegrity structure where struts are modeled with linear elastic mass-tensor and cables with viscoelastic Kelvin-Voigt elements.

\section{B. Formulation of finite element model "Linear elastic Mass Tensor model for Tensegrity model}

In this section, we introduce the fundamental concepts for description and measurement of the deformation using finite element. Firstly we introduce briefly the relation between stress tensor $\sigma$ and strain tensor $\varepsilon$ (Hooke's law), then we present the model mass-tensor for tensegrity model.

1) Linear elastic model: The strain tensor characterizes the change in shape (or deformation) near a material point. For the large deformations this tensor called GreenLagrange is spelt:

$$
\mathbf{L}=\frac{1}{2}\left(\operatorname{grad}(\underline{U})+\operatorname{grad}^{T}(\underline{U})+\operatorname{grad}(\underline{U}) \cdot \operatorname{grad}^{T}(\underline{U})\right)
$$

For the small deformations, we can write the infinitesimal strain tensor :

$$
\boldsymbol{\varepsilon}=\frac{1}{2}\left(\operatorname{grad}(\underline{U})+\operatorname{grad}^{T}(\underline{U})\right)
$$


The Cauchy stress tensor $\sigma_{i j}$ represents internal forces acting at a material point per unit area of the deformed solid. The Newton's law of conservation of linear momemtum becomes in terms of the Cauchy stress tensor:

$$
\underline{\operatorname{div}}(\boldsymbol{\sigma})+\underline{f}=\rho \underline{U}
$$

2) Hooke's Law or "Hookean" materials: Define a relation between the stress tensor and the strain tensor. This law for isotropic material and for linear deformation can be written:

$$
\boldsymbol{\sigma}=\lambda \operatorname{tr}(\boldsymbol{\varepsilon}) \mathbf{I}+2 \mu \boldsymbol{\varepsilon}
$$

where $\mu$ : shearing coefficient and $\lambda$ : compression coefficient are the Lame's constants.

3) Mass-Tensor finite element formulation: In this section we present different steps for the linear elastic mass-tensor model introduced by [22][25] and extended by [26]. In our work, the struts of tensegrity model are the volume object, these struts are meshed with 3D first order tetrahedral element Fig.6. The displacement field $\underline{U}(\underline{x})$ is defined by a linear interpolation function of the displacement vectors at each vertex $\underline{u}^{j}$ of the 4 vertices of the tetrahedron $T^{k}$ as follows: (5).

$$
\underline{U}(\underline{x})=\sum_{j=0}^{3} N^{j}(\underline{x}) \underline{u}^{j}
$$

where $N^{j}(\underline{x})$ are linear interpolation functions ; these shape functions are simply the tetrahedral barycenter coordinates.

Introducing $\underline{P}^{i}$ the coordinates of each tetrahedron vertex and the areas vector $\underline{m}^{i}$, the general expression of displacement field in the tetrahedron can be defined by:

$$
\underline{U}(\underline{x})=\frac{-1}{6 V^{T}} \sum_{j=0}^{3} \underline{m}^{j} \cdot\left(\underline{x}-\underline{P}^{j+1}\right) \underline{u}^{j}
$$
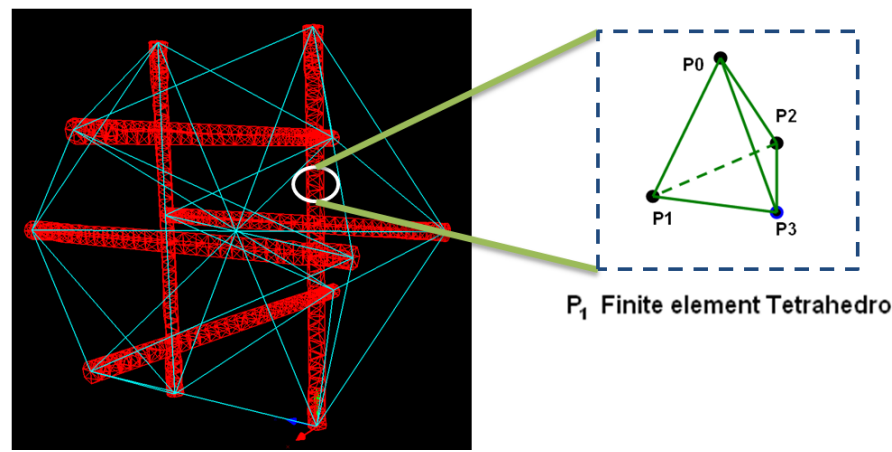

Fig. 6. Using the linear tetrahedron element for the struts of the tensegrity model.

We can express the stored energy in the tetrahedron $T^{k}$ as a function of the displacement of the 4 vertices and the Lame's constants as follows:

$$
W=V^{T}\left[\frac{\lambda}{2}(\operatorname{tr}(\boldsymbol{\varepsilon}))^{2}+\mu \operatorname{tr}(\boldsymbol{\varepsilon})^{2}\right]
$$

Finally, the force $\underline{f}^{i}$ applied to the summit $i$ of a tetrahedron $T^{K}$ is obtained as the partial derivative of the strain energy with respect to associated nodal displacement :

$$
\underline{f}^{i}=-\frac{\partial W}{\partial \underline{u}^{i}}=\sum_{j=0}^{3} \mathbf{K}^{i j} \cdot \underline{u}^{j}
$$

where $\mathbf{K}^{i j}$ : are the elements stiffness matrix, these tensors depend on the rest geometry of the $T^{K}$ and Lames coefficients:

$$
\mathbf{K}^{i j}=\frac{1}{36 V^{K}}\left(\lambda \underline{m}^{i} \otimes \underline{m}^{j}+\mu \underline{m}^{j} \otimes \underline{m}^{i}+\mu \underline{m}^{i} \cdot \underline{m}^{j} \mathbf{I}\right)
$$

The equation (8) defines the force $f^{i}$ for one tetrahedron $T^{K}$, but for global mesh, we must add the contribution by all adjacent tetrahedra of $T^{K}$, the resulting force $\underline{F}^{i}$ can be expressed as follows :

$$
\underline{F}_{m e s h}^{i}=\mathbf{K}^{i i} \cdot \underline{u}^{i}+\sum_{j \in O\left(P^{i}\right)} \mathbf{K}^{i j} \cdot \underline{u}^{j}
$$

$\mathbf{I K}^{i i}$ and $\mathbf{I K}^{i j}$ design respectively the sum of tensor $\mathbf{K}^{i i}$ associated with the tetrahedra adjacent to node $i$ and with the tetrahedra adjacent to edge $(i, j), O\left(P^{i}\right)$ is the neighborhood of vertex $P^{i}$. These tensors, depending only on the rest geometry and Lames coefficients, are constant and can be pre-computed. It's the essential advantage of the method of mass-tensor which makes it usable for real time application.

\section{Cable behavior for the tensegrity structure}

In this section, we present the description of the cable behavior about our tensegrity model (Fig.5). These cables are assumed to be have as viscoelastic mass-spring-damper. Each cable is modeled with two masses interconnected via spring and damper in parallel (Voigt-Kelvin model, Fig.7). In the local frame, the relation between the stress and the strain can be written as follows:

$$
\sigma=E \varepsilon+\eta \dot{\varepsilon}
$$

with $\sigma=\frac{F}{S}$ and $\varepsilon=\frac{l-l^{0}}{l^{0}}$, where $F$ : is the applied load in the extremity of the cable, with $l$ and $l^{0}$ and $S$ are respectively the resting length of the cable, the initial length and the section of the cable. we replace these parameters in (11), we obtained :

$$
\underline{F}_{\text {local }}^{c a b}=\left(\frac{E S\left(l-l^{0}\right)}{l^{0}}+\frac{\eta \dot{l}}{l^{0}}\right) \underline{x}
$$

In the global coordinates, for the tensegrity structure, we can expand this equation easily and obtained the force applied in each node by summing the forces contributed by all nodes connected (Fig.7), this equation can be written as follow:

$$
\underline{F}_{i}^{c a b}=\sum_{j}\left\{\left(E S\left(\frac{\left|\underline{p}_{i j}\right|}{L_{i j}}-1\right)+\eta\left(\frac{\underline{v}_{i j} \cdot \underline{p}_{i j}}{L_{i j}\left|\underline{p}_{i j}\right|}\right)\right) \frac{\underline{p}_{i j}}{\left|\underline{p}_{i j}\right|}\right\}
$$




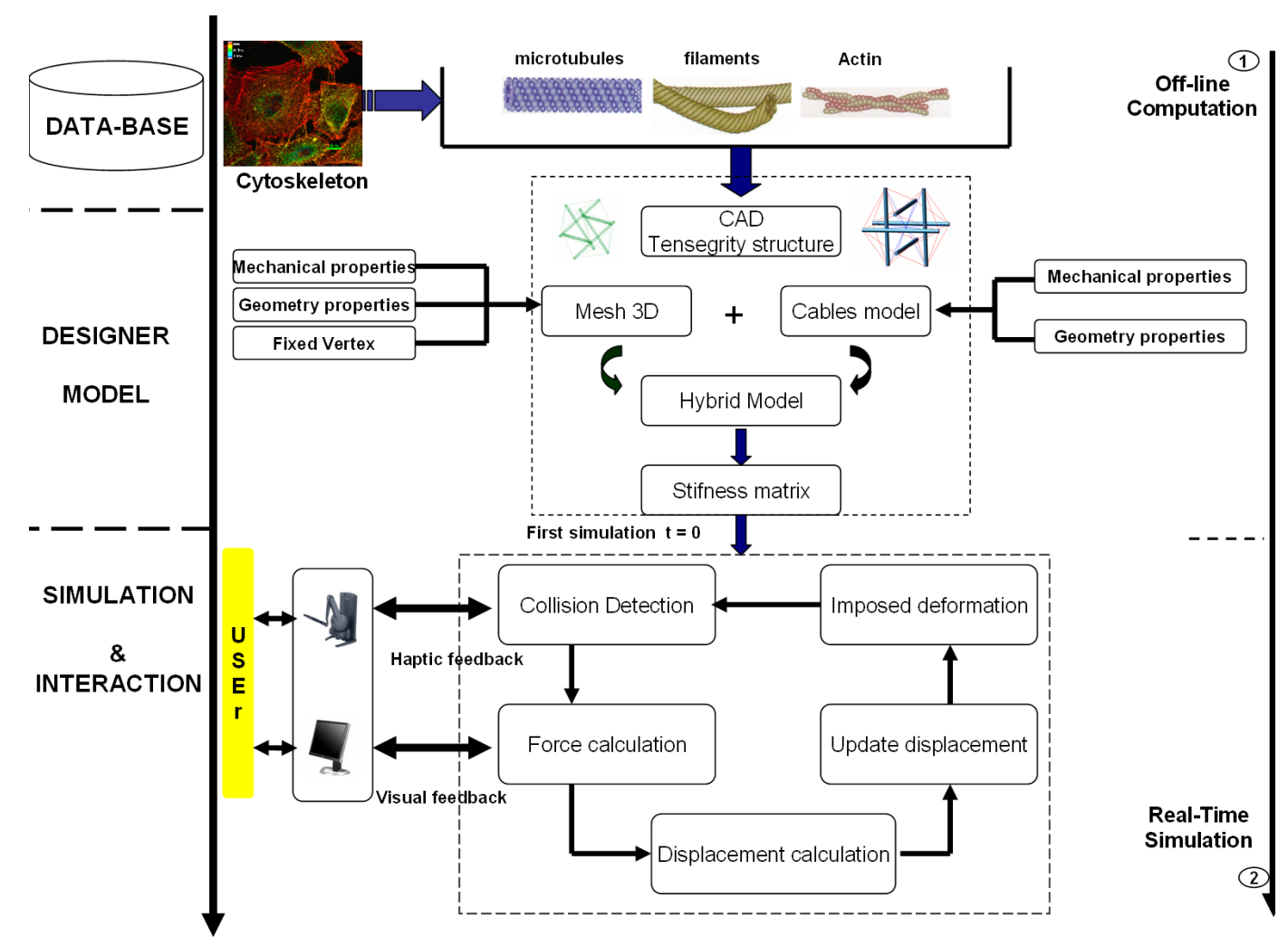

Fig. 8. Structure of the simulation of the cell injection. The figure shows the two phases used for the simulation in real time. off-line calculations and real time simulation phase used the haptic feedback and visual feedback

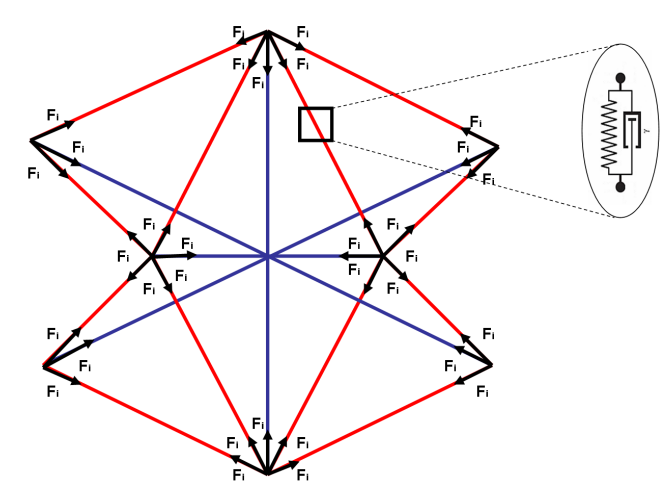

Fig. 7. Mass-spring-damper model for cable structure.

therefore, these cables do not transmit the forces in compression, in this case we can introduce a Heavyside function:

where:

$$
H(x)=\left\{\begin{array}{l}
0 \text { si } x \\
1 \text { si } x \geq 0
\end{array}\right.
$$

$$
\begin{aligned}
& \underline{F}_{i}^{c a b}=\sum_{j}\left\{\left(H ( | \underline { p } _ { i j } | - l _ { i j } ^ { 0 } ) \left(E S\left[\frac{\left|\underline{p}_{i j}\right|}{l_{i j}^{0}}-1\right]\right.\right.\right. \\
& \left.\left.+\eta\left(\frac{\underline{v}_{i j} \cdot \underline{p}_{i j}}{l_{i j}^{0}\left|\underline{p}_{i j}\right|}\right)\right) \frac{\underline{p}_{i j}}{\left|\underline{p}_{i j}\right|}\right\}
\end{aligned}
$$

The force applied to an extremity of the struts of the tensegrity structure, is the sum of the forces exerted by the struts and the cables (Fig.7).

1) Dynamic Model For the Tensegrity model: We suppose that the mass of the cell is lumped at the vertex. The dynamic law of motion can be written for a vertex $i$ as:

$$
M^{i} \underline{\ddot{U}}^{i}+\gamma \underline{\dot{U}}^{i}=\underline{F}^{i}
$$

where $M^{i}$ is the mass and $\gamma$ a numerical structural damping coefficient.

Many different techniques can be used to construct time integration computational algorithms. We choose a classical second order central finite difference scheme as a good compromise between simulation in real time and accuracy:

$$
\underline{O P}^{i}(t+h)=K_{1}\left(\underline{F}^{i}(t)+K_{2} \underline{O P} \underline{P}^{i}(t)-K_{3} \underline{O P}^{i}(t-h)\right)
$$

where $h$ is the time step; $\left\{\begin{aligned} K_{1} & =\frac{h^{2}}{M^{i}+\gamma^{i} h} \\ K_{2} & =\frac{2 M^{i}+\gamma_{i} h}{h^{2}} \\ K_{3} & =\frac{M^{i}}{h^{2}}\end{aligned}\right.$

\section{A ViRTUAL ENVIRONMENT SYSTEM FOR CELL INJECTION TRAINING}

\section{A. System hardware and software}

In a real time virtual reality based cell simulation system, the operator would be able to interact with the threedimensional model of cell using his sense of vision as well 
TABLE I

MATERIAL PROPERTIES ASSIGNED TO THE CELLULAR COMPONENTS IN EACH MODEL [18][19][27]

\begin{tabular}{|c||c|c|c|}
\hline & Microfilaments & Microtubules & Intermediate filaments \\
\hline \hline $\mathrm{E}(\mathrm{Pa})$ & $2.6 \times 10^{9}$ & $1.2 \times 10^{9}$ & $0.3 \sim 0.4 \times 10^{9}$ \\
\hline$v$ & 0.3 & 0.3 & 0.3 \\
\hline $\mathrm{D}(\mathrm{nm})$ & $5 \sim 9$ & 25 & $\simeq 10$ \\
\hline Section $\left(\mathrm{nm}^{2}\right)$ & 18 & 190 & - \\
\hline
\end{tabular}

as actively manipulate using his sense of touch. Fig.8 shows the description of the VR based real time cell injection trainer. Usually, real time graphics translates to an update rate of $30 \mathrm{~Hz}$, stable haptic interaction requires as much higher update rate $(1 \mathrm{kHz})$. As soft biological tissues exhibit complex viscoelastic and nonlinear properties, the real time interaction imposes severe restrictions on the cell tensegrity model. A significant difficulty of using the finite element technique for real time simulation is that it is computationally costly. These mesh-based schemes also require an expensive numerical integration operation for the computation of the system stiffness matrices. For this reason we adopted the following computational procedure:

1) Off-line computation: : The proposed computational architecture is composed of an off-line pre-calculation step. All the operations costly in time are realized during this step. The database contains all the models composing the biological cytoskeleton structure, i.e. the microtubules, filaments and actins components. The designer assembles the different components to form a tensegrity structure through a CAD MSC-software (MARC-ADAMS). The meshing of the tensegrity structure is then carried out through a dedicated 3D meshing software GID software. We adopted tetrahedron elements with fixed number of nodes (see section Fig.6). The mechanical and geometrical properties of the tensegrity structure are settled in Table.I where $E, v$ and $D$ are respectively the Young modulus, Poisson ratio and diameter for these filamentous of the cytoskeleton. Finally, we added to the initial structure some cable models connected at the extremities of the struts with given mechanical boundary properties (pre-stressed conditions). We initiated then off-line calculation of all tensors $K_{i j}$ and we stocked the precalculated stiffness values.

2) On-line simulation: : Since haptics and graphics have different update frequencies, we have implemented separate threads to update the loops. During the injection task, the contact between tool and cell must occur at a special set of points (nodal points). It results in an imposed displacement of the tensegrity structure at these loading nodes. Therefore, to prevent loss of resolution, the density of nodal points should be sufficiently high leading to an increase in the problem size. The collision detection module specifies the type of tool-tissue interaction. The tool in virtual environment can be modeled as a complex 3D object, composed of numerous surfaces, edges and vertices but it constitutes a challenging task. In our implementation, we have used a point-based representation of the pipette injector and have employed a simple ray-triangle intersection with local search technique [28]. The visual feedback is updated after each displacement calculation step and the haptic feedback is returned after each force calculation step.

\section{SIMULATION}

The mesh that has been used for the following simulations are shown in Fig.6. It is constituted by a non regular tetrahedron volume mesh composed of 1014 vertices and 1798 tetrahedra where all vertices are free and only three nodes are fixed (see Fig.10). In these figures, we show preliminary simulations of tensegrity deformation during biological cell injection. In this case, we tested two configurations. Firstly, the penetration axis of the micropipette is aligned with the tensegrity geometrical center. The force of the micropipette is applied to the center of one strut of the tensegrity model Fig.9. Secondly, the penetration axis of the micropipette is parallel to the first load but the applied loads are located in the extremity of the strut Fig.10. We can observe clearly the deformation of the tensegrity structure. These results shows also the influence of the penetration axis with respect to cell deformation when considering a complete model (biomembrane + cytoskeleton + cytoplasm ). As illustration, the Fig.11 shows the nonlinear behavior of the cell deformation and punction. These results are in good agreement with the experimental results obtained on injected oocytes cells by J.D Desai in [29] and B.J. Nelson in [30].
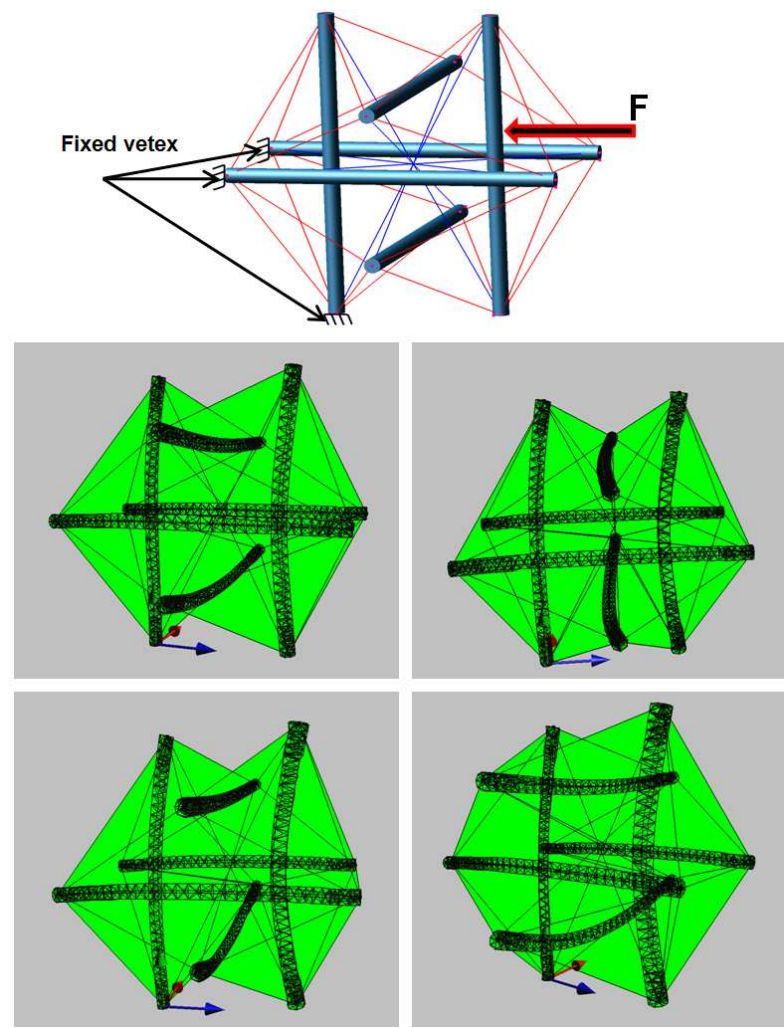

Fig. 9. Configuration 1: The force of the micropipette is applied to the center of one strut. 

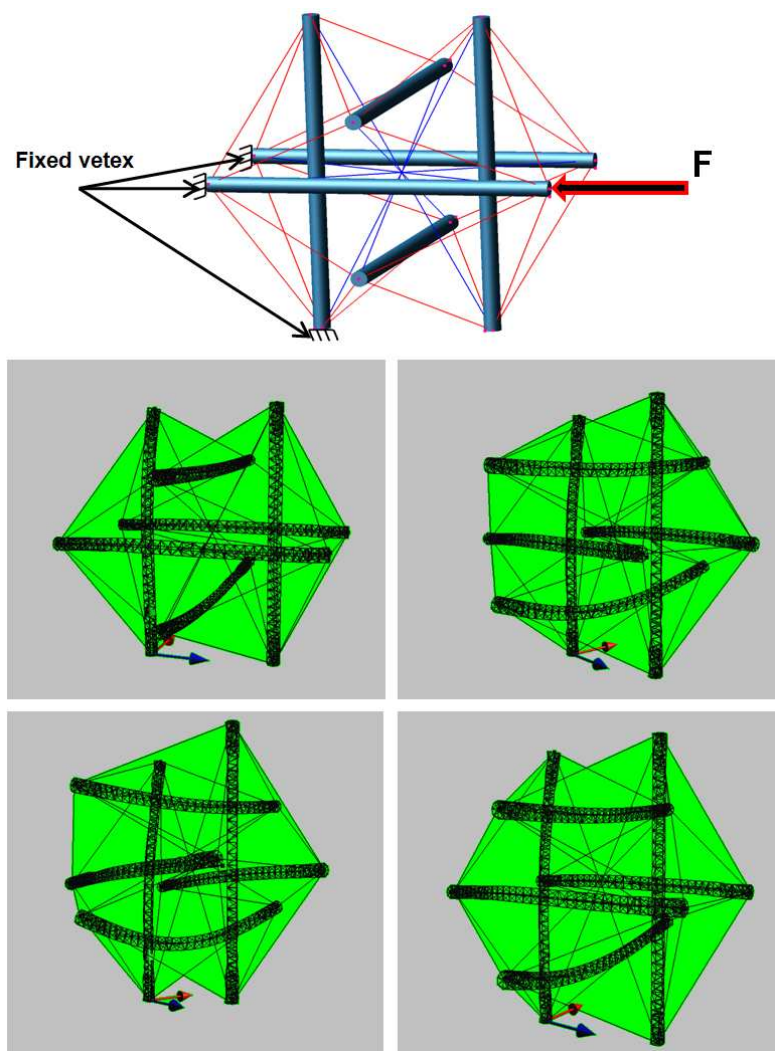

Fig. 10. Configuration $2:$ The force of the micropipette is applied in the extremity of the strut.

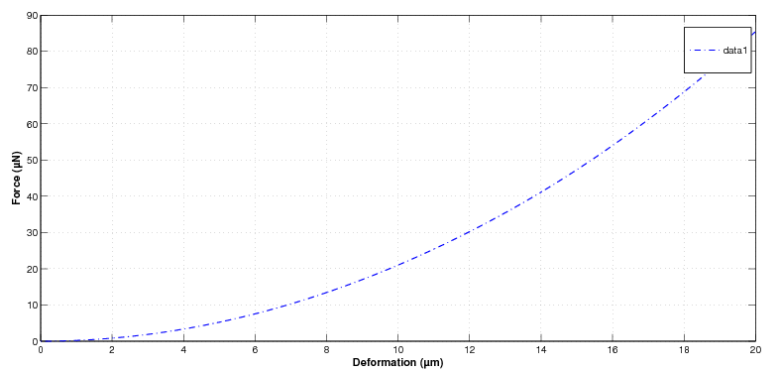

Fig. 11. Force as function of displacement.

\section{Discussion AND CONCLUSION}

The objectives of this work were to propose a virtual environment for a ICSI-based simulator. Using 3D graphics and force feedback device, an unexperienced operator can interact with a virtual deformable object with its own sense. We first investigated the challenging issues in the modeling of the bio-mechanical properties of living cells through tensegrity models. Then, the finite element simulations showed clearly that the proposed dynamic model is able to simulate the cell deformations in real time. However, the preliminary results point out different limitations. First, it does not take into account the nonlinear and viscoelastic behavior of the cytoplasm during insertion. The biomembrane bounded by the pellucida zona and cytoplasm will be divided into tetrahedrons and mass points arranged at each vertex of tetrahedrons. Second, injection operation implies that the topological deformation should use real time vertex reallocation of the meshing. We are planning to integrate an iterative relaxation algorithm which can adapt its mesh resolution during punction of the cell membrane (generation of a hole when the pipette passes the bio-membrane). Third, the validity of the deformation and reaction force during injection procedures have not been verified. These different research goals are under investigation.

\section{REFERENCES}

[1] Abolhassani N., Patel R. and Moallem M., Needle insertion into soft tissue : A survey, Med Eng Phys, 29 (4), pp. 413-431, 2007.

[2] Evans E., A.Yeung A., Apparent viscosity and cortical tension of blood granulocytes determined by micropipet aspiration, Biophys J 56, pp. 151-160, 1989.

[3] Schmid-Schnbein G. W. , Sung K. L., H Tzeren H., Skalak R., and Chien S., Passive mechanical properties of human leukocytes, Biophys J., 36(1), pp. 243-256, 1981.

[4] Mow V. C., Kuei S. C., Lai W.M. and Armstrong C. G., Biphasic creep and stress relaxation of articular cartilage in compression: theory and experiments, Journal of Biomechanical Engineering, 102, pp. 73-84, 1980.

[5] Delingette H. and Ayache N., Soft tissue modelling for surgery simulation, In N. Ayache, Ed. Computational Models for the Human Body: Handbook of Numerical Analysis, Elsevier, 2004.

[6] Desai J. , Pillarisetti A., and Brooks A. D., Engineering Approaches to Biomanipulation, Annual Review of Biomedical Engineering, 9, pp. 35-53, 2007.

[7] Dumoulin J.M. Coonen, E., Bras M., Bergers-Janssen J.M., IgnoulVanvuchelen R.C., van Wissen L. C.P. et al., Embryo development and chromosomal anomalies after ICSI effect of the injection procedure, Hum Reprod. 16, pp. 306-312, 2001.

[8] Ebner T. ,Yaman C. , Moser M., Sommergruber M., Jesacher K. and Tews G., A prospective study on oocyte survival rate after ICSI influence of injection technique and morphological features, J Assist Reprod Genet 18, pp. 601-606, 2001.

[9] Kim J.,Choi C., De S. and Srinivasan M. A. , Virtual surgery simulation for medical training using multi-resolution organ models, Int J Med Robotics Comput Assist Surg, 3, pp. 149-158, 2007

[10] Satava R. M., Surgical education and surgical simulation, World J Surg, 25(11), pp. 1484-1489, 2001.

[11] Stamenovic D. ,Ingber D.E. , Models of cytoskeletal mechanics and adherent cells. Biomechanics and Modeling in Mechanobiology 1(1), 2002, pp: 95-108.

[12] Wang N., Naruse K., Stamenovic D. , Fredberg J.J., Mijailovich S.M. Tolic-Norrelykke I.M., Polte T., Mannix R., Ingber D.E., Mechanical behavior in living cells consistent with the tensegrity model, Proc Natl Acad Sci USA 98 (2001) 7765-77703.

[13] Stamenovic D., Fredberg J.J., Wang N., Butler J.P., Ingber D.E.,A microstructural approach to cytoskeletal mechanics based on tensegrity, Journal of Theoretical Biology 181 (2), pp. 125-136, 1996.

[14] Ingber D.E. , Tensegrity: the architectural basis of cellular mechanotransduction, Annual Review of Physiology, 59 (1), pp. 575-599, 1997.

[15] Svitkina T.M. , Verkhovsky A.B., Borisy G.G., Plectin sidearms interaction of intermediate filaments with microtubules and other components of the cytoskeleton, J Cell Biol 135, pp. 991-1007, 1996.

[16] Lim C. T.,. Zhou E. H, Quek S. T., Mechanical models for living cells - A review, Journal of Biomechanics, 39, 2, 195-216, 2006.

[17] Sultan C. ,Stamenovic D. , Ingber D. E., A computational tensegrity model predicts dynamic rheological behaviors in living cells, Annals of Biomedical Engineering. Vol.32, No.4, pp.520-530, 2004.

[18] McGarry J. G., Prendergast, $A$ three-dimensional finite element model of an adherent eukaryotic cell, European Cells and Materials, Vol. 7, pp.27-34, 2004.

[19] Janmey P. A, Euteneuer U., Traub P., and Schliwa M. Viscoelastic properties of vimentin compared with other filamentous biopolymer networks, Journal Cell Biologie, volume 113, pp 155-160, 1991.

[20] Canadas, P. V., M. Laurent, C. Oddou, D. Isabey, and S.Wendling.Acellular tensegrity model to analyze the structural viscoelasticity of the cytoskeleton. J. Theor. Biol. 218, pp. 155-173, 2002 . 
[21] Meier U, Lopez O, Monserrat C, Juan M.C. and Alcaniz M., Real-time deformable models for surgery simulation: a survey, Comput Methods Programs Biomed, 77(3), pp. 183-197, 2005.

[22] Bro-Nielsen M. and Cotin S., Real-Time Volumetric Deformable Models for Surgery Simulation Using Finite Elements and Condensation, Computer Graphics Forum (Eurographics), 5(3), pp. 57-66, 1996.

[23] Debunne G. , Desbrun M., Cani M. P.and Barr A., Dynamic RealTime Deformations Using Space and Time Adaptive Sampling, Proc. Siggraph, ACM Press, pp. 31-36, 2001.

[24] Picinbono J. , Delingette H., Ayache N., Non-linear and Anisotropic Elastic Soft Tissue Models for Medical Simulation,. Proc, IEEE International Conference on Robotics and Automation, Seoul, Korea, 1370-1375, 2001.

[25] Cotin S. ,Delingette H., Ayache N., A Hybrid Elastic Model for RealTime Cutting, Deformations, and Force Feedback for Surgery Training and Simulation, Visual Computer 16, 437-452, 2000.

[26] Schwartz J.-M. Dellinger, M.,Rancourt D. ,Moisan C. and Laurendeau D. , Modeling Liver Tissue Properties Using a Non-linear ViscoElastic Model for Surgery Simulation, Medical Image Analysis, 9(2), pp. 103-112, 2005

[27] Wagner O.I., Rammensee S., Korde N., Wen Q., Leterrier J.F. and Janmey P.A., Softness, strength and self-repair in intermediate filament networks, Experimental Cell Research ,313(10), pp. 2228-2235, 2007.

[28] Moller T. and Trumbore B., Fast, Minimum Storage Ray-Triangle Intersection, journal of graphics tools, vol. 2, no. 1, pp.21-28, 1997.

[29] Pillarisetti A. ,Pekarev M. , Brooks A. D., Desai J.P., Evaluating the Effect of Force Feedback in Cell Injection, IEEE Transactions on Automation Science and Engineering, 4(3), pp. 322-331, 2007.

[30] Sun Y., Wan K., Roberts K. P., J. C. Bischof J.C. and Nelson B. J., Mechanical property characterization of mouse zona pellucida, IEEE Transactions On NanoBioscience, 2(4), pp. 279 - 286, 2003.

[31] Boal D., Mechanics of the Cell. Cambridge University Press,Cambridge, 2002.

[32] M. Ammi, A. Ferreira, Biological Cell Injection Visual and Haptic Interface: Advanced Robotics, Vol.20, N0.3, 2006.

[33] R. Alterovitz, et al.: Planning for steerable bevel-tip needle insertion through 2D soft tissues with obstacles. IEEE international conference on robotics and automation (ICRA). 2005. p. 16527.

[34] HW, Nienhuys, AF. van der Stappen: A computational technique for interactive needle insertions in 3D nonlinear material. IEEE international conference on robotics and automation (ICRA). 2004. p. 20617. 